\title{
SAMINBOT: UN ASISTENTE VIRTUAL PARA RECOLECTAR DATOS DURANTE LA PANDEMIA DEL COVID-19
}

\author{
HARLEY VERA OLIVERA \\ harleyve@gmail.com / ORCID ID 0000-0003-2011-8797 \\ Ana Rocío Cárdenas Maita \\ ar.cardenasmaita@usp.br / ORCID ID 0000-0001-9879-3229 \\ Meluni Daney Palomino Flores \\ meluni.palomino.flores@gmail.com / ORCID ID 0000-0002-9171-0619 \\ Jonathan Ricardo Vasquez ChICATA \\ jrvasquez54@gmail.com / ORCID ID 0000-0002-9295-5685 \\ ROSA VIRGINIA ENCINAS QUILLE \\ rosavirginiae@gmail.com / ORCID ID 0000-0001-9166-1741 \\ Grover ENRIQUE CASTRO GUZMÁN \\ grover.org.rf@gmail.com / ORCID ID: 0000-0002-2900-3235 \\ YANINA LEON USCAPI \\ yaninaleonuscapi@gmail.com / ORCID ID: 0000-0003-2388-5342 \\ JHONY LUCIA HUALLPARIMACHI GARCÍA \\ jhony.huallparimachi@upr.edu / ORCID ID 0000-0002-1150-3661 \\ LUIS ANTONIO QUisPe CARTAgena \\ luis.2qc@gmail.com / ORCID ID 0000-0001-5256-5431 \\ LAURO ENCISO RODAS \\ lauro.enciso@unsaac.edu.pe / ORCID ID 0000-0001-6266-0838 \\ Departamento Académico de Ingeniería Informática \\ Universidad Nacional de San Antonio Abad del Cusco, Perú
}

\section{Resumen}

La falta de base de datos para tomar decisiones en acciones rápidas durante la pandemia ocasionada por el COVID-19 mostró la necesidad de usar nuevas tecnologías para agilizar el proceso de captura de información descentralizada. Este artículo presenta un asistente 
virtual (chatbot) denominado SaminBot, como una alternativa para recolectar datos y brindar información durante la pandemia del COVID-19. Este chatbot se aplicó en la región del Cusco, en el Perú, con conversaciones en las áreas de salud, economía y educación entre enero y agosto del 2020. SaminBot inicia la recolección de datos en función del área de interés del usuario, obtiene información demográfica del mismo y lo va guiando a través de preguntas con la intención de proveerle información útil de acuerdo con su situación personal. Los cuestionarios fueron validados por especialistas de acuerdo con su campo, el proceso de recolección de datos inició en enero y finalizó en junio del 2021 mediante las plataformas WhatsApp, Facebook Messenger y la página web donde se obtuvo 1586 registros.

PALABRAS CLAVE: chatbot / salud / educación / economía / SARS-CoV-2 /, COVID-19 coronavir / pandemia Cusco

\section{SAMINBOT: A VIRTUAL ASSISTANT TO COLLECT DATA DURING THE COVID-19 PANDEMIC}

\section{Abstract}

The lack of datasets to make decisions in quick actions during the pandemic caused by COVID-19 showed the need to use new technologies to speed up the process of capturing decentralized information. In this article, we present a virtual assistant (chatbot) called SaminBot, as an alternative to collect data and provide information during the COVID-19 pandemic; this chatbot opperated in the Cusco region of Peru with conversations in the areas of health, economy, and education from January to August 2020. "SaminBot" starts data collection based on the user's area of interest, obtains demographic information, and guides him through questions to provide helpful information according to his situation. Specialists validated the questionariess. Data gathering was performed from January to June 2021 through WhatsApp and Facebook Messenger platforms and the project's website, where 1586 records were obtained.

KEYWORDS: chatbot / health / education / economy / SARS-CoV-2 / COVID-19 /

/ Ccoronavir / Cusco pandemic 


\section{INTRODUCCIÓN}

Uno de los grandes desafíos durante la época de la pandemia causada por el COVID-19 en los años 2020 y 2021 (Ciotti et al., 2020) fue la necesidad de soluciones tecnológicas para dar soporte al proceso de toma de decisiones (Bentlage et al., 2020; Haug et al., 2020). Los gobiernos y universidades tomaron acciones estableciendo ciertos planes para contener, retrasar, investigar y mitigar (Adolph et al., 2021; GOV.UK, 2020; Guidon y Amato, 2020; Shadmi et al., 2020). Al mismo tiempo, uno de los mayores obstáculos cuando se toma en cuenta a los sistemas automatizados que ayudan en la toma de decisiones es la falta de datos (datasets) actualizados y de rápida recolección que estén disponibles para ser usados por la comunidad científica (He et al., 2021). Datos que nos ayuden, por ejemplo, a predecir sucesos de gran importancia como nuevos brotes de enfermedades, planear cómo lidiar con los problemas no relacionados con la salud causados por la pandemia, y/o ofrecer buenas medidas de la magnitud de un brote rodeando alguna ubicación específica. En relación con esto, países en vías de desarrollo como el Perú sufrieron consecuencias colaterales debido a estas necesidades, al punto que no existía un fácil acceso a fuentes confiables de información que pudieran ayudar a planear procesos de atención de los acontecimientos producidos en el país. Una de las plataformas de datos abiertos es "Perú en tus manos" (GOB.PE, 2020) con más de un millón de usuarios, pero estos datos no son suficientes para poder hacer análisis eficientes en las diferentes áreas afectadas, como salud (Chatterjee et al., 2020; Giraldo, 2020), educación (Figueroa et al., 2021; Tarkar, 2020) y economía (Borio, 2020; Varona y Gonzales, 2021). Áreas en las que el país fue afectado por la pandemia. Como respuesta a este problema, se propuso una solución tecnológica para recolectar información que refleje la situación actual de los ciudadanos del país lo más fielmente posible, especialmente en zonas del interior del país como el Cusco.

Una barrera inmediata para la recolección de información fue el relativo aislamiento que desfavorece al intercambio de información. Por lo tanto, medios como encuestas presenciales, visitas programadas o centros físicos de información quedan totalmente descartados como fuentes de obtención de datos. Así, la comunicación masiva ya existente por medio de internet se convierte en un potencial medio de obtención de datos. Actualmente, la mensajería instantánea (MI) es el medio de comunicación más difundido (Jennings et al., 2006; Stieger y Göritz, 2006) y el que menos conocimiento especializado y recursos económicos necesita para ser utilizado por una persona promedio. Sin embargo, la naturaleza poco formal de la Ml implica un sistema de intercambio de información muy desorganizado y difícil de procesar en cantidades masivas. Muchos tipos de aplicaciones de servicio al cliente, como los sitios web de comercio electrónico, usan chatbots para comunicarse con sus clientes; estos se están volviendo populares. Un chatbot o agente conversacional, es decir, un sistema que puede interactuar o chatear con un usuario humano en lenguaje natural (lo y Lee, 2017) 
Este proyecto consideró los beneficios de expansión y fácil alcance a las personas en tiempos de cuarentena que ofrece la MI (Besolí et al., 2018; Kaufmann y Peil, 2020). Se optó por usar esta herramienta para la divulgación de un conjunto de cuestionarios especializados que abarcaron temas centrales entre los problemas más críticos durante la pandemia. Se realizaron cuestionarios en las áreas de salud, economía y educación. Dichos cuestionarios fueron el resultado de un trabajo conjunto con especialistas en dichas áreas quienes durante varios meses brindaron su asesoramiento para seleccionar las preguntas que mejor pudieran medir el impacto del COVID-19 en la población. Tales cuestionarios fueron distribuidos masivamente por medio de chatbots embebidos en las redes sociales de Facebook, Whatsapp y un sitio web creado específicamente para el proyecto. Además, junto con las preguntas de los cuestionarios se presentó información relacionada con el tema de la pregunta para poder informar sobre temas relacionados que podrían ayudar al encuestado a sobrellevar de mejor forma la pandemia. Esto último sirvió también como motivo de difusión de su utilidad en un contexto tan cambiante como el de la pandemia. Esta también es una característica que lo diferencia de otros medios de recolección de datos tradicionales; en el anexo A se pueden observar ejemplos de las conversaciones en las tres áreas por las redes mencionadas.

Para evitar la variabilidad en las respuestas dadas por los usuarios, se aplicó un cuestionario con respuestas fijas (elegidas en conjunto con los especialistas de cada área) en lugar de permitir que los individuos pudiesen dar repuestas abiertas. Otro beneficio de esta medida es que la única información que es compartida por el entrevistado es información que se predefinió como relevante por especialistas en las áreas de interés. Cabe mencionar que ya existen soluciones tecnológicas para la recopilación de información por medio de internet y MI (García Brustenga et al., 2018; Herrero-Diz y Varona-Aramburu, 2018; Muhammad et al., 2020; Rosruen y Samanchuen, 2018). Sin embargo, la aplicación y métodos utilizados no tienen el objetivo de conectar con diversas partes de una población (por ejemplo., distritos de Cusco con docentes, alumnos, profesionales de salud y población en general) y diversas áreas (salud, educación y economía) a través de un mismo chatbot. Por esta diversidad el chatbot debe cambiar de personalidad conforme al usuario que está interactuando (por ejemplo, con un docente la conversación es más formal y con un adolescente es más coloquial).

El foco de la información que se deseaba obtener incluye mayormente, una compilación de los problemas que eran enfrentados diariamente por la población de la ciudad de Cusco a causa de la pandemia. En el área de salud, se buscó obtener información sobre los hábitos de movilización de las personas, hábitos de higiene e historial con la COVID-19 (por ejemplo, cuántos miembros de la familia del entrevistado fueron contagiados con la enfermedad). En el área de economía, se buscó información sobre problemas financieros que puedan estar atravesando las personas y los negocios (por ejemplo, si tuvieron que cerrar temporalmente o definitivamente un negocio por la falta de demanda). En el 
área de educación, se buscó conocer sobre los problemas educativos ocasionados por la situación actual (por ejemplo, cómo ha cambiado el rendimiento de alumnos de una cierta edad al no haber más clases presenciales).

El proyecto se centró en la población de la región Cusco, localizada en el sur del Perú. A fin de tener más cercanía con la población cuzqueña, el chatbot tomó el nombre de SaminBot, y fue representado por el personaje que se aprecia en las conversaciones de las figuras 2 y 4. Este artículo detalla cada uno de estos puntos en las próximas secciones. El trabajo se divide de la siguiente forma. En la segunda sección, Trabajos relacionados, se presenta trabajos relacionados al uso de chatbots como herramienta para la recolección de datos. En la tercera sección, Metodología, se presenta la metodología de investigación adaptativa que se siguió en el proyecto. En la cuarta sección, Desarrollo y arquitectura, se presentan las bases tecnológicas para la construcción del chatbot. En la quinta sección, Instrumentos y método de la investigación, se presenta una descripción básica de los cuestionarios preparados para las tres áreas. En la sexta sección, se presentan los resultados más destacados del proyecto. Finalmente, las conclusiones del trabajo se presentan en la séptima sección.

\section{TRABAJOS RELACIONADOS}

Se realizaron muchos esfuerzos para combatir los nuevos desafíos impuestos por la pandemia generada por el COVID-19. Los chatbots han resultado ser una herramienta útil en la solución de diversos problemas, principalmente por el fácil uso y acceso por parte de los usuarios, además del distanciamiento social requerido en tiempos del COVID-19. En el sector salud, el chatbot presentado por Judson et al. (2020) fue utilizado para evaluar y buscar síntomas o exposiciones al COVID-19 entre los trabajadores de salud de la Universidad de San Francisco antes de iniciar las jornadas laborales. De esta forma, el uso del chatbot redujo los tiempos de espera de los empleados que ingresan a los hospitales durante los cambios de turno. Además, este proceso ha permitido el distanciamiento físico en las entradas de los hospitales y el cuidado de personas con alto riesgo de contagio en el centro de salud. El chatbot implementado es de fácil acceso a través de su un aplicativo propio y el sistema interno del centro de salud.

En el trabajo de Bharti et al. (2020), se propuso el chatbot conversacional denominado Aapka Chikitsak, con el objetivo de brindar servicios de telesalud en la India. El chatbot fue implementado utilizando el servicio en nube Google Cloud Platform (GCP). Mediante este servicio se ofrece a los pacientes acceso a información sobre atención médica. Mediante el uso de este tipo de tecnología, los autores buscan aprovechar el potencial de la inteligencia artificial para cerrar la brecha en la demanda y la oferta de proveedores de atención médica humana en zonas rurales de la India. El chatbot demostró un alto potencial en la reducción de barreras de acceso a centros de salud mediante consultas inteligentes de forma remota, ayudando así de manera efectiva a la sociedad. 
Para servicios de atención telefónica y registro de casos potenciales, el chatbot propuesto por Martin et al. (2020) consiguió identificar casos del COVID-19 utilizando un reporte de síntomas. El chatbot, denominado Symptoma, es un asistente digital que puede diferenciar entre más de 20000 enfermedades con una precisión de más de $90 \%$. Para el caso del COVID-19, Symptoma consiguió reconocer la enfermedad con una precisión de 96,32 \%. Para esto, el sistema utiliza un conjunto de casos clínicos combinados con informes de casos del COVID-19. Además, Symptoma permite la entrada de texto libre, complementada con preguntas de seguimiento específicas de la enfermedad, en 36 idiomas.

Viendo la importancia de los chatbots producidos para combatir el COVID-19, (Schubel et al., 2021) propuso el análisis de 69451 pacientes contactados vía SMS o correo electrónico. Se analizaron los datos demográficos e información sobre las interacciones de los participantes que fueron recolectados mediante un chatbot que proporcionó un módulo de aprendizaje y un evaluador de síntomas. El análisis muestra diferencias en el uso de las funciones del chatbot por género, raza y edad. Esta información podría informar mejor las estrategias de difusión de información de salud pública para el COVID-19.

Si bien es cierto existen chatbots propuestos para combatir el COVID-19 de distintas formas, fue necesario un análisis detallado sobre la posibilidad de la aplicación de esta tecnología en nuestro medio considerando características y actitudes específicas de nuestra población. Aspectos como el acceso a internet, el distanciamiento social y la familiaridad con la tecnología fueron considerados.

\section{METODOLOGÍA}

El asistente virtual SaminBot fue el resultado de un proyecto de investigación adaptativa desarrollado de agosto de 2020 a setiembre de 2021. El objetivo de ese proyecto fue validar una herramienta tecnológica de asistencia virtual como instrumento para recolección de datos y que al mismo tiempo provea información a la población durante la pandemia causada por el COVID-19, periodo en el que hubo mucha incertidumbre y restricciones debido a la cuarentena. Para alcanzar tal objetivo se siguieron las siguientes etapas:

\subsection{Diseño de los cuestionarios.}

Las preguntas de los cuestionarios de recolección de datos se plantearon usando la técnica de encuesta con los participantes. Se buscó que las preguntas del cuestionario fueran lo más didácticas y breves posible, dentro de lo permitido por una herramienta tecnológica del chatbot y manteniendo siempre el formalismo necesario para que las respuestas sean objetivas y rápidas. También se usaron recursos adicionales como imágenes, infografías, textos explicativos, consejos breves, emoticones, entre otros para capturar la atención de los usuarios. 
En tal sentido, todas las preguntas y la información adicional que se entregó al usuario pasaron por varios procesos de análisis y revisión del equipo de investigación junto con los profesionales especialistas en las áreas mencionadas anteriormente. Los especialistas trabajaron desde el inicio del proyecto; ellos actuaron como consultores externos y apoyaron al equipo de investigación en todo el proceso de desarrollo del cuestionario desde la elaboración y validación de las preguntas hasta las pruebas de funcionamiento del chatbot antes de que este fuese publicado.

\subsection{Implementación del asistente virtual}

Se implementó una arquitectura tecnológica que permitió que los scripts del chatbot sean embebidos en plataformas de redes sociales populares, por medio de las cuales se popularizó en la población cuzqueña. Para la implementación de esta arquitectura se siguió una metodología de desarrollo de software ágil. En la etapa del despliegue del chatbot se realizaron varias pruebas controladas para evaluar tanto la calidad en software como la aceptación de los usuarios. Para evaluar la calidad de las preguntas por cada sector se escogieron personas aleatoriamente y con ellos se realizó una simulación de los cuestionarios y una prueba de aceptación. Con los resultados obtenidos de esa simulación logramos verificar la calidad de las preguntas y su aceptación por parte de los participantes en un medio digital. Adicionalmente, para evaluar la calidad del software se usó el estándar internacional ISO-25 010. Asimismo, dicho modelo proporciona a los actores del software un entorno flexible que puede ser adaptado al desarrollo de chatbots. También se logró evaluar el chatbot en las ocho características de calidad: adecuación funcional, eficiencia de desempeño, compatibilidad, usabilidad, fiabilidad, seguridad, mantenibilidad y portabilidad. Debido a que este artículo pretende mostrar las lecciones aprendidas con todo este proceso de implementación y despliegue del chatbot, en la cuarta sección se describe con más detalle la arquitectura implementada.

\subsection{Aplicación de los cuestionarios}

El chatbot denominado SaminBot del proyecto estuvo en línea por seis meses, desde enero a junio del 2021. Durante este periodo se realizaron campañas publicitarias por redes sociales para captar la mayor cantidad de usuarios y así lograr que estos lo conozcan e interactúen con él. Se planearon estrategias de difusión como: (i) una página web donde se publicó información general sobre el proyecto; (ii) una página de Facebook donde se publicaban imágenes y videos relacionados a la pandemia con información oficial que era atrayente para los usuarios; (iii) se creó al personaje SaminBot, el cual tuvo un logotipo y personalidad cercana a la población cuzqueña.

La interacción con el chatbot iniciaba con el saludo por parte de una persona al robot por medio de cualquiera de las vías disponibles (Facebook, WhatsApp y página web), ante esto SaminBot se presentaba y mostraba las cláusulas de anonimato de los datos, una 
vez que la persona aceptaba dichas cláusulas, ésta era derivada a un menú donde podía escoger el tema de su preferencia para ser encuestado. Durante la encuesta y según las respuestas de la persona se le fue prestando infografías y consejos útiles relacionados al tema puntual de la pregunta. A pesar de que hubo muchas interacciones con el chatbot, no todas las personas terminaron de llenar los cuestionarios, esto debido a las limitaciones tecnológicas que posee un robot en función a la intención de las personas. A pesar de ello se logró tener un total de 1586 usuarios que interactuaron con el chatbot en las tres áreas de salud, economía y educación con encuestas validas.

La población objetivo para la aplicación del cuestionario fueron los ciudadanos cuzqueños mayores de 18 años. Los individuos debían contar con dominio básico en el uso de cualquiera de las herramientas tecnológicas como WhatsApp, Facebook o páginas web, y con acceso a internet en algún momento del día. En el caso de las encuestas para educación se consideró una población de estudiantes de secundaria mayores de 15 años.

Los criterios que se utilizaron para la elección de las preguntas en los cuestionarios contaron con la intervención y validación de especialistas en cada área, como se puede observar un ejemplo ilustrativo en la figura 1. Estos fueron planteados realizando un estudio de diferentes instrumentos y métodos en cada área para poder recopilar el estado de la población.

- En el área de salud, se buscó obtener información sobre los hábitos de movilización de las personas, hábitos de higiene e historial con el COVID-19; de esta forma se utilizó el cuestionario COVID Impact Survey realizado por NORC en la Universidad de Chicago, que rastrea el impacto del COVID-19 en la salud (Wozniak et al., 2020).

- En el área de economía, se buscó información sobre problemas financieros que puedan estar atravesando las personas y los negocios, se consideró como base la encuesta de medición de capacidades financieras en los países andinos publicada por Mejía et al. (2015).

- En el área de educación, se buscó conocer sobre los problemas educativos ocasionados por la situación actual (Unidad de Gestión Educativa Local Puno, 2020).

Todos los flujos del proceso de conversación fueron clasificadas en cuatro grupos: flujo de inicio de conversación, flujo de economía, flujo de salud y flujo de educación. En el tabla 1 se lista los cuestionarios desarrollados en Saminbot para cada área. En la figura 2, la interfaz principal de usuario para iniciar una conversación con Saminbot. Los datos brutos de la recopilación de estos datos y los cuestionarios con las respectivas preguntas se encuentran disponibles en Zenodo'.

1 https://doi.org/10.5281/zenodo.5630360 


\section{DESARROLLO Y ARQUITECTURA}

Esta sección presenta la arquitectura utilizada para el desarrollo de SaminBot; ofrece una descripción detallada de cada componente utilizado. La arquitectura para el desarrollo de Saminbot (figura 3) tuvo como base la arquitectura de chatbots e inteligencia artificial propuesto por Dion Hinchcliffe (2016).

(a)
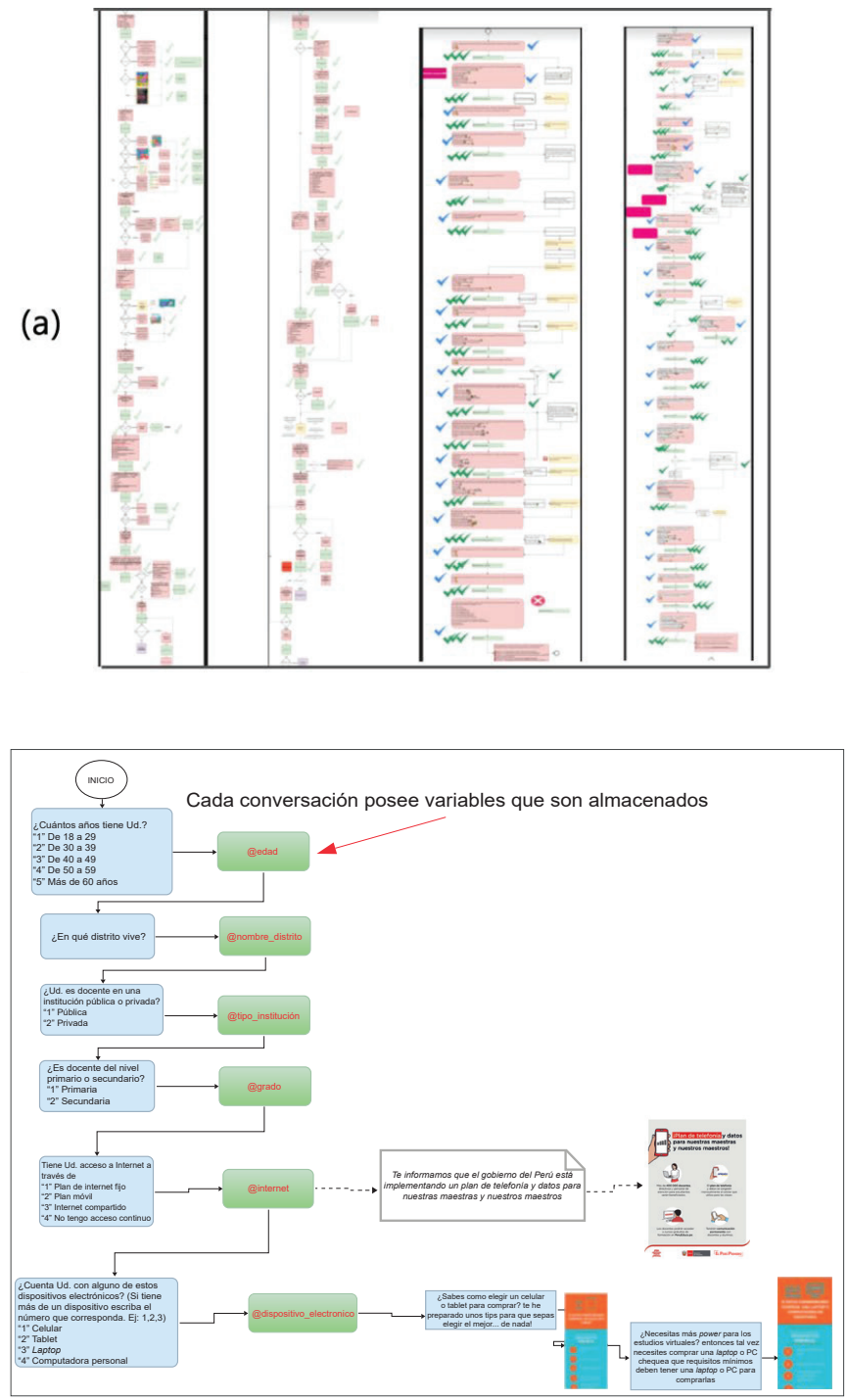

Figura 1. Validación de las encuestas de cada área: (a) Desarrollo y revisión de diagramas de flujo para todas las áreas. (b) Ilustración de un fragmento del diagrama de flujo de Educación, note que se insertó variables para cada uno de las respuestas obtenidas de los usuarios.

Elaboración propia 
Tabla 1

Clasificación de flujos para interacción con los usuarios

\begin{tabular}{ll}
\hline Flujo & Descripción \\
\hline (1) Flujo de inicio de conversación & Formas en que el usuario llegará al chatbot y cuestionario \\
& de caracterización general. \\
(2) Flujo de economía & Cuestionario economía familiar. \\
& Cuestionario economía de empresas. \\
& Informativo económico. \\
(3) Flujo de salud & Cuestionario prevención. \\
& Cuestionario diagnóstico y tratamiento. \\
(4) Flujo de educación & Cuestionario para el docente. \\
& Cuestionario para el estudiante. \\
& Cuestionario para el tutor de familia. \\
\hline
\end{tabular}

Elaboración propia

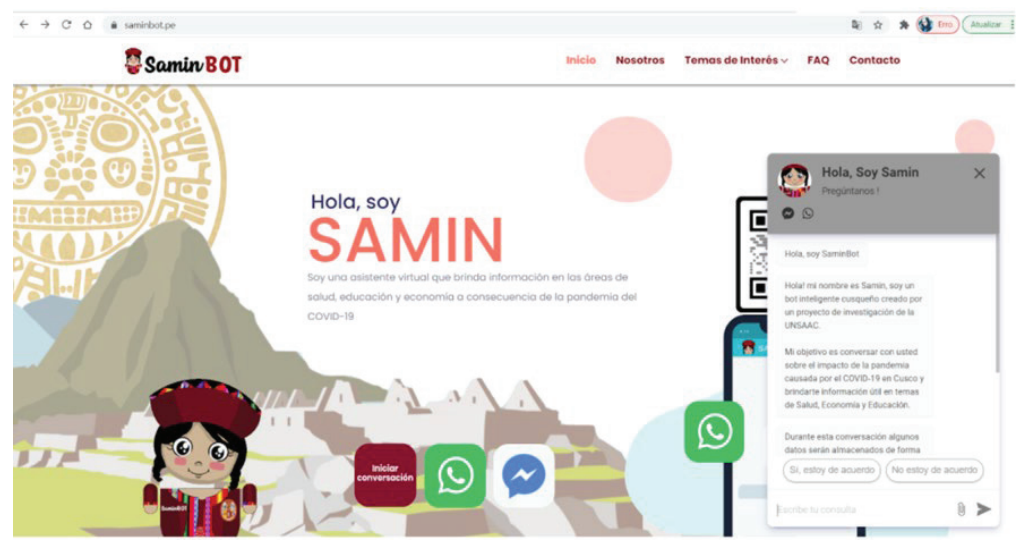

Figura 2. Presentación de interfaz de asistente virtual Samintbot de recolección de datos en tiempos de pandemia del COVID-19.

Elaboración propia 

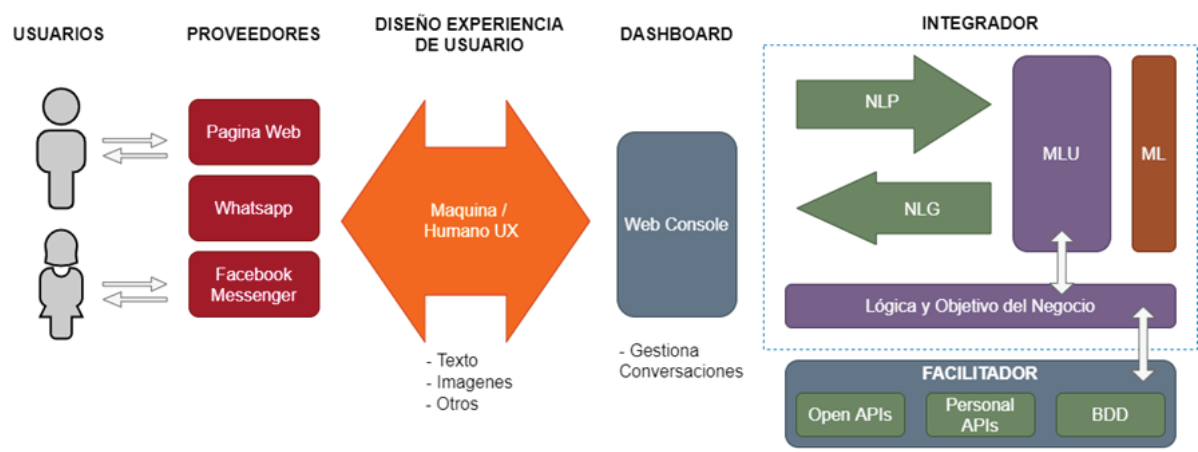

Figura 3. Arquitectura general de Saminbot

Elaboración propia

La presente arquitectura muestra todas las capas que intervienen dentro de Saminbot. Comienza con el usuario enviando un mensaje a Saminbot a través de un canal, a este canal lo llamaremos proveedor, que es la interfaz por la cual el usuario interactúa con el chatbot. Los proveedores que usamos para Saminbot son tres y estas se ilustran en la figura 4; la página web oficial del proyecto, Whatsapp, Messenger y Facebook Messenger. Siguiendo hacia la derecha nos encontramos con la capa de UX, que se divide en dos UX Interface y UX Writting. UX Interface responde a la forma en la que se va a mostrar el contenido dentro del canal y está diseñada por el proveedor, por ejemplo Whatsapp y su interfaz mediante su aplicación móvil o interfaz web (Whatsapp web). UX Writting se encarga de ver como Saminbot se comunica con el usuario, a través de textos, entidades, links, botones, flujos (figura 1), contextos, intenciones, eventos, parámetros, imágenes, etc. todas las herramientas que permiten responder al usuario.

En la capa de dashboard se ha integrado una aplicación web que sirve de intermediario entre la capa de UX y la capa integrador; esta capa permite guardar todas las interacciones de todos los usuarios con Saminbot de todos los proveedores, así podremos visualizar en tiempo real las conversaciones que vienen desde la página web, Whatsapp y Facebook Messenger. Además, permite contestar mensajes desde la consola y permite descargar las conversaciones en formato .csv para su posterior análisis. Llegamos al Integrador, la parte más importante de Saminbot, que contiene todas herramientas de NLP y machine learning. Para Saminbot, la capa de integración está a cargo de DialogFlow que se encarga de todo el proceso de NLP, NLU, ML y NLG. NLP es Natural Language Processing, que es el proceso que realiza el chatbot para adquirir, identificar e interpretar el lenguaje humano. Una vez procesado se pasa a otro proceso llamado NLU o Natural Language Understanding y su trabajo consiste en identificar la frase que el usuario envió, es decir comprender el significado de la intreacción. 


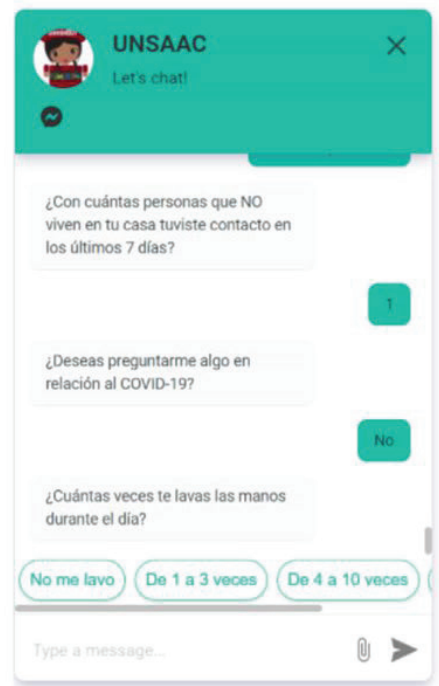

(a)

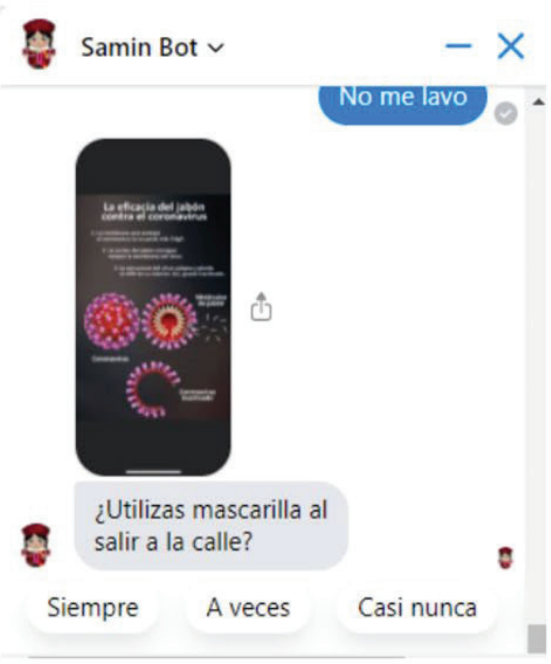

(b)

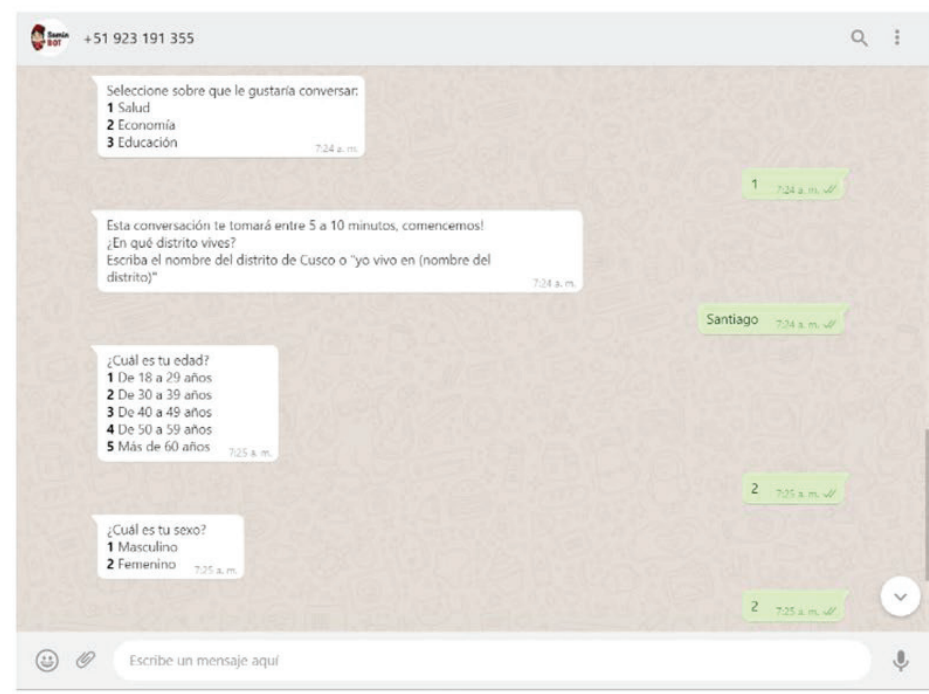

(c)

Figura 4. Interfaces asistente virtual Saminbot: (a) Interfaz proveedor web, los usuarios son identificados por un ID de usuario generado automáticamente al iniciar la conversación, conversación adecuada al proveedor, por ejemplo las alternativas mediante botones. (b) Interfaz proveedor Facebook Messenger, los usuarios son identificados por el usuario Facebook y la interfaz de conversación es similar al proveedor web. (c) Interfaz proveedor WhatsApp, los usuarios son identificados por el número de WhatsApp y la interfaz de conversación es diferente a los otros proveedores, por ejemplo, las alternativas son mediante números.

Elaboración propia 
Una intención por parte del usuario es la razón por la cual se quiere comunicar con Saminbot. Las intenciones fueron registradas en la plataforma de DialogFlow y están construidas en un formato de preguntas y respuestas según los criterios y validaciones de los especialistas de salud, educación y economía. DialogFlow al ser un producto de Google, tiene soporte para español y ofrece un buen rendimiento para los procesos de NLP, NLU, ML o machine learning, es decir identifica las intenciones de manera correcta y se entrega la respuesta; esta respuesta es generada como un natural language generation (NGL), este proceso permite generar lenguaje natural a través de una respuesta preprogramada específica para cada intención identificada en la plataforma de DialogFlow. En la parte inferior tenemos al facilitador, que en este caso es un servidor de base de datos que se comunica con el integrador y, dependiendo del objetivo de Saminbot por cada sector de interés (salud, educación, economía), almacena datos sobre el COVID-19 en formato de texto o imágenes, y que el integrador usa para mostrar las respuestas a las intenciones de los usuarios. El facilitador también es usado para guardar las respuestas de los usuarios que interactuaron con Saminbot en conformidad con la Ley de protección de datos personales N. 29733 del Perú.

\section{DISENO DE LOS INSTRUMENTOS}

Esta sección presenta una descripción detallada de los cuestionarios preparados para la recolección de datos en las áreas de salud, economía y educación. También, se describe el procedimiento utilizado para la recolección de los datos.

\subsection{Cuestionario de salud}

Como medida de mitigación en el área de salud en la región del Cusco se desarrolló un cuestionario que, además de recabar datos en el área de salud, también proveía información relevante de fuentes confiables (Ministerio de Salud, Organización Mundial de la Salud y en Centro de Control de Enfermedades - USA). El cuestionario fue desarrollado en conjunto con una especialista epidemióloga. Este cuestionario fue dividido en cuatro secciones (1) Caracterización general de la población, (2) Medidas de prevención del COVID-19, así como también preguntas sobre el (3) diagnóstico y tratamiento del COVID-19.

La caracterización de la población consistió en 9 preguntas y obtiene datos generales como el estatus socio-económico, datos generales de la población y distribución geográfica. La parte de prevención tiene doce preguntas sobre las medidas de prevención que la persona usó para prevenir el COVID-19; y, finalmente, la parte de diagnóstico contiene quince preguntas de las que se obtienen datos sobre el diagnóstico y tratamiento del usuario con COVID-19. El cuestionario recaba información como el número de familiares afectados por el COVID-19, los síntomas persistentes y la duración de los síntomas en las personas diagnosticadas con COVID-19. 


\subsection{Cuestionario de economía}

El impacto de la pandemia en el Cusco ha perjudicado a cientos de familias, microempresas y empresas, causando que muchas empresas cierren o busquen formas de reinventarse. Por otro lado, la mayoría de estudios económicos sobre el comportamiento de la pandemia se centralizaron en la capital, Lima. Al momento de realizar la revisión bibliográfica inicial de este proyecto, no se encontró información suficiente para realizar estudios específicos sobre datos económicos en la región Cusco.

En este proyecto se plantearon dos grupos de preguntas para el área de economía; el primer grupo referido a la economía familiar de las personas y cómo fueron afectadas en ese aspecto, y el segundo grupo referido a la economía de los microempresarios y microemprendedores del Cusco, ya que la mayor parte de los negocios en la región están en estos grupos. El cuestionario de economía familiar tuvo como objetivo evaluar el estado económico de las familias, así como los aspectos que fueron más afectados por las restricciones debidas a la cuarentena por la pandemia del coronavirus. Al formular las preguntas para este cuestionario se consideraron los siguientes criterios: (a) conocimientos básicos sobre gestión de un presupuesto familiar; (b) disponibilidad de dinero para necesidades básicas durante la pandemia; (c) necesidad de crédito y cumplimiento de los requisitos para acceder a dicho crédito; (d) capacidad de pago de créditos y otras cuentas básicas; (e) Actitudes frente al uso del dinero y la empleabilidad dentro del hogar durante la pandemia.

Así mismo, el cuestionario de economía en negocios tuvo como objetivo evaluar el estado económico de los microempresarios y microemprendedores con negocios locales en todos los rubros. Se buscó evaluar en qué aspectos se vieron más afectados por las restricciones dadas y la crisis económica causada por la pandemia del COVID-19. Al formular las preguntas para este cuestionario se consideraron los siguientes criterios: (a) manejo de la economía de los negocios; (b) niveles de producción; (c) problemas que afectaron la comercialización; (d) niveles de empleabilidad; (e) actitudes frente al manejo de las finanzas; y, (f) acceso a programas de ayuda lanzados por el gobierno específicamente para cubrir las necesidades de los microemprendedores.

\subsection{Cuestionario de educación}

Así como en muchos países latinoamericanos, la educación en Perú ha sido afectada de forma severa por la pandemia. En lo que respecta a educación, el gobierno peruano decidió suspender las clases presenciales para pasar a las sesiones de aprendizaje en línea en las escuelas públicas y privadas. Este contexto educacional plantea nuevos desafíos para mantener el normal desarrollo de las clases y estándar educativo del país. Estos desafíos involucran aspectos relacionados con la nueva tecnología utilizada, el impacto psicológico en los distintos actores y el factor económico que afecta este proceso de adaptación. 
Por lo tanto, contar con datos confiables sobre estos aspectos y proporcionados directamente por los actores involucrados es muy importante para comprender plenamente la situación real de la educación. Se prepararon tres cuestionarios dirigidos a: (a) profesores de primaria y secundaria, (b) estudiantes de secundaria mayores de 13 años, y (c) padres de familia y/o tutores. Los cuestionarios utilizados en el estudio fueron adaptados a las características educativas de esta región Cusco.

Los cuestionarios para profesores y estudiantes se organizaron de la siguiente manera: (a) preámbulo, cuyo propósito es caracterizar a los participantes, (b) tecnología, para recolectar datos relacionados al proceso de adaptación de profesores y estudiantes a los nuevos dispositivos y plataformas virtuales en el nuevo contexto educativo, (c) psicología, para recopilar datos relacionados con la salud mental y el impacto psicológico que la educación remota provocó en los actores, y finalmente (d) otras preguntas, para recopilar datos adicionales. El cuestionario para padres también incluye preguntas de preámbulo similares a las utilizadas para profesores y estudiantes. En este cuestionario se incluyen también un conjunto de preguntas de opinión sobre el desempeño de los profesores y el funcionamiento de las plataformas virtuales en el proceso educativo a distancia. El cuestionario para padres y tutores se basa en el cuestionario para padres implementado por la Unidad de Gestión Educativa de Moho, con respecto a la plataforma de aprendizaje en línea Aprendo en Casa, aplicada por el gobierno peruano a nivel nacional (Unidad de Gestión Educativa Local - Puno, 2020).

Estas preguntas fueron recolectadas en entrevistas a docentes y con la ayuda de una especialista en el área de educación y psicología educativa se adaptaron estas preguntas a un lenguaje cercano al docente para el caso del cuestionario dirigido a docentes; de forma similar se procedió con el cuestionario con los alumnos, con quienes inclusive se usó emoticones provistos por la red social. Se identificaron los indicadores más importantes en cada área y las preguntas para medir dichos indicadores. En total, se prepararon veintiocho preguntas para profesores, dicinueve para alumnos y dicienueve para padres y tutores. Para validar el correcto funcionamiento de los cuestionarios se realizaron pruebas a través de entrevistas realizadas por miembros del equipo de investigación y por los desarrolladores del chatbot.

\section{RESULTADOS}

En esta sección se describe los resultados de la aplicación de la encuesta para caracterizar a los entrevistados y los resultados que destacan más para los cuestionarios aplicados por sectores. 


\subsection{Descripción de resultados generales}

Las preguntas generales fueron en relación al sexo, grupo étnico, nivel educativo y distrito donde vive. A continuación, se presenta una descripción de la información reportada por los entrevistados.
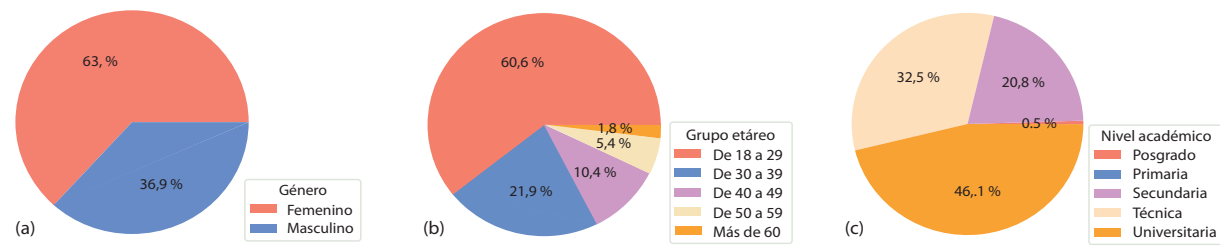

Figura 5. Caracterización general de la población alcanzada: (a) Población encuestada según género. (b) Población encuestada según edad. (c) Población encuestada según nivel académico.

Elaboración propia

Con relación al sexo, el 63,1 \% fueron mujeres y el otro $36,9 \%$ fueron hombres, como se observa en la figura 5 (a), la herramienta tuvo una notoria acogida del grupo femenino.

Así mismo, a pesar de la difusión generalizada del chatbot por medio de redes sociales, como era de esperarse por tratarse de una tecnología moderna, ésta tuvo más acogida por la población joven, entre 18 y 29 años, con un 60,6 \% del total de personas que realizaron algún tipo de interacción. El siguiente grupo con mayor acogida fue la población adulta joven, entre 30 y 39, años con un 21,9\%, seguido del grupo de 40 a 49 años con un 10,4 \%. Finalmente, las personas mayores a 50 años suman 7,2 \%. En la figura 5 (b) se observa esta distribución.

Otra segmentación en los datos obtenidos es con relación al grado de instrucción, según las respuestas de las personas, el 46,1 \% de la población entrevistada cuenta con educación universitaria, el 32,5\% cuenta con educación superior técnica, el 20,8 \% cuenta solo con secundaria y el $0,6 \%$ restante corresponde personas con posgrado 0 primaria. En la figura 5 (c) se observa esta distribución.

Finalmente, en los datos obtenidos en relación a la localidad donde viven, según las respuestas de las personas el 73,8 \% pertenecen a la provincia del Cusco y el 7,5 \% pertenecen a otras provincias del Cusco. Resultados con respuestas de otras localidades a nivel nacional fueron considerados como otros. En la figura 6 se observa esta distribución. 


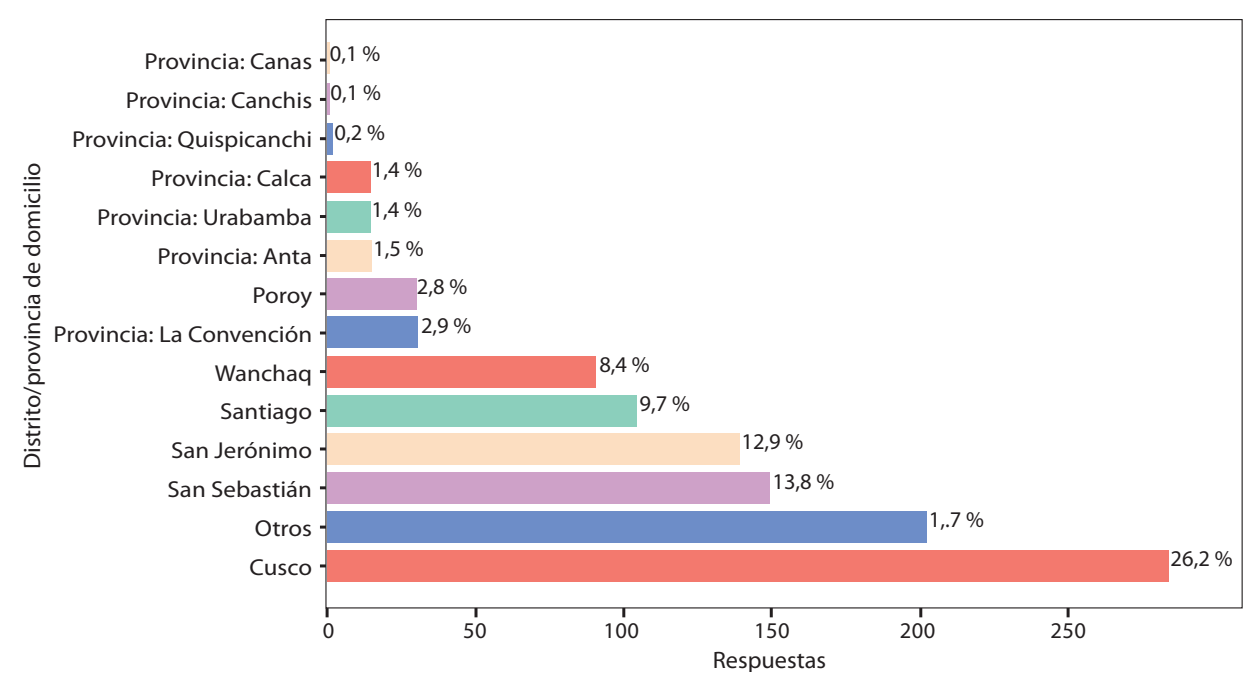

Figura 6. Población encuestada según lugar de residencia.

\section{Elaboración propia}

En resumen, los datos recolectados por esta encuesta corresponden a una población mayormente femenina, entre 18 y 50 años, que cuenta con educación superior ya sea universitaria o técnica. Cabe destacar que tales distribuciones pueden variar según el tipo de encuesta con la que cada persona interactuó. Algunas personas interactuaron con el chatbot en las tres áreas, salud, economía y educación (sin redundancia de información) y otras lo hicieron en apenas una de ellas según su preferencia.

\subsection{Descripción de resultados por sector}

\subsection{1 Área de salud}

La casi inexistente articulación entre los diferentes prestadores de servicios de salud debilitó la respuesta de los servicios sanitarios y ocasionó un impacto mayor en la población más pobre y vulnerable. Por ello, en el área de salud decidimos focalizar la encuesta en: (1) prevención. Se obtuvieron datos sobre el cumplimiento de las indicaciones dadas por el Ministerio de Salud para prevenir el contagio como el lavado de manos, uso de mascarilla, medio de transporte, motivos de salida a la calle, contacto con personas, actividades canceladas debido al COVID-19 y la automedicación contra el COVID-19. En este cuestionario también se incluyen detalles sobre la frecuencia del lavado de manos, uso de mascarilla, salida a la calle, tipo de transporte utilizado, entre otros. Asimismo, en (2) diagnóstico y tratamiento, se obtuvo los siguientes datos de cada participante: diagnóstico COVID-19, tipo de prueba COVID-19, fecha de diagnóstico, tipo de tratamiento recibido, síntomas persistentes del COVID-19 y número de familiares fallecidos con 
COVID-19. Se recolectaron datos de 527 individuos en todos los cuestionarios del sector salud. Del total de individuos que interactuaron con el chatbot $77,8 \%(410)$ son mujeres y $22,2 \%$ (117) son varones. El $60,2 \%$ con edades entre 18 a 29 , el $24,8 \%$ con edades entre 30 a 39 mientras que el resto tiene más de 40 años. Cabe resaltar que en el área de salud se exigió una secuencia estricta y que todas las respuestas fuesen respondidas (de acuerdo a los flujos de conversación).

\subsection{2 Área de economía}

En el área de economía, los cuestionarios buscaron evaluar el comportamiento y las actitudes de las personas en dos grupos: (1) Encuesta de economía familiar; aquí se obtuvo datos sobre la realización de un presupuesto tomando en cuenta ingresos y gastos, conocimiento de productos financieros, acceso a crédito durante la pandemia, dificultades para obtener un crédito, pagos crediticios, conductas y actitudes frente al dinero, frecuencia del manejo de estados de cuenta, impacto económico por familia, actitudes frente a las dificultades de la pandemia, reacción en cuanto a la pérdida de ingresos o empleo, medidas de aislamiento social que perjudicaron al entrevistado, remodelación de viviendas, adquisición de propiedades y tipos bonos que beneficiaron a los cuzqueños durante la pandemia. Por otro lado, (2) Encuesta de economía para negocios, se obtuvieron datos para tipo de negocio, si el negocio es formal o informal, ubicación del negocio por distritos, asociatividad a algún grupo de pequeños empresarios, comparación del nivel de producción antes y durante la pandemia, problemas que enfrentaron los negocios en la pandemia, si el negocio optó por trabajar remotamente, suspensión o despido de trabajadores, negocios que quebraron, rango de meses en los que se cerraron, negocios que realizaron un cambio de rubro, venta o traspasos de maquinaria, venta o traspaso de locales o inmuebles, dificultades para conseguir un financiamiento, sobrendeudamiento, remodelación de negocios, adquisición de locales comerciales, opinión en relación a los programas de reactivación para negocios brindados por el gobierno durante la pandemia.

De la población que interactuó con el chatbot 51 \% son mujeres, el 61 \% están entre 18 a 29 años, el 73,8 \% son de la provincia del Cusco. Las interacciones con el cuestionario de economía familiar llegaron a 386, entre las estadísticas a respuestas por pregunta se destaca que el $46,8 \%$ no maneja ningún tipo de producto financiero; existe la actitud de comprar solo en caso de necesidad en un 94,2 \% de los entrevistados y $62,8 \%$ tiene actitud de ahorro; hubo pérdida de ingresos familiares en un $81,1 \%$; y la falta de trabajo afectó a un 31,6 \% de los entrevistados. Las interacciones con el cuestionario de economía de negocios fueron de 493, entre las estadísticas a respuestas por pregunta a los dueños de negocios, se destaca que 65,7 \% están en la informalidad; $82,2 \%$ no tiene ningún tipo de asociatividad con negocios similares; los problemas que más afectaron a los negocios fueron la disminución de la demanda (25,4\%), restricciones del estado de 
emergencia (15\%), competencia informal (14\%), abastecimiento insuficiente de materias primas e insumos (14\%); y la percepción de quiebra del negocio fue del $86,2 \%$ con gran mayoría en los meses de marzo y abril del 2020. Cabe destacar que en los cuestionarios para el área de economía no se exigió una secuencia estricta ni de la obligatoriedad de responder a todas las preguntas, por tal motivo cada pregunta tiene independencia en las respuestas y no está en correlación con otras.

\subsection{3 Área de educación}

Uno de los desafíos más importantes de la implementación de la educación a distancia en el Perú fue la capacitación de docentes y estudiantes para el uso de las herramientas tecnológicas necesarias para llevar a cabo las actividades escolares. El estudio buscaba conocer las sensación de docentes y estudiantes respecto a su condición en el uso de estas herramientas. El chatbot desarrollado para educación consta de tres cuestionarios dirigidos a docentes, estudiantes, padres, madres y/o tutores. Este proceso se llevó a cabo a través de numerosas reuniones en las que se identificaron los indicadores más importantes en cada área y las preguntas para medir dichos indicadores, como: (1) Capacitación: en forma general es posible observar que la percepción de los docentes para las instituciones públicas es de estar medianamente capacitados, en comparación con los docentes de instituciones privadas que tienen una sensación de estar muy capacitados en el uso de herramientas digitales. Otro punto interesante es la sensación de los alumnos de no haber recibido ningún tipo de capacitación, no obstante lo bajo de estos porcentajes, ningún docente reportó no haber recibido capacitación para el uso de herramientas digitales. (2) Conectividad: docentes y estudiantes fueron consultados sobre algunos aspectos relacionados a la conectividad. Las respuestas sugieren que los docentes, tanto de instituciones públicas como privadas, cuentan con un acceso aceptable a internet. En el caso de los estudiantes, es posible ver que el acceso disminuye, sobre todo en los estudiantes de instituciones públicas, quienes reportaron no tener acceso continuo a internet. (3) Motivación: el Ministerio de Educación lanzó el 2020 la estrategia de educación a distancia Aprendo en Casa dirigida a estudiantes que permanecen en sus viviendas a causa de la cuarentena. Padres y madres de familia y/o tutores de estudiantes perciben en su mayoría estar de acuerdo con el programa de Aprendo en Casa. Existe una mayor cantidad de encuestados de sexo femenino que monitorea el aprendizaje de sus hijos y/o apoderados. (4) Ambiente de estudio: En relación a los factores de distracción que enfrentan los estudiantes, es posible observar que las redes sociales son predominantes tanto para estudiantes de instituciones públicas o privadas. Según las respuestas de los estudiantes, otro factor de distracción es la familia, esto se observa sobre todo en estudiantes de instituciones públicas. Sobre el ambiente en el que los estudiantes reciben sus aulas virtuales, una mayoría respondió utilizar un cuarto propio sin diferencias entre estudiantes de instituciones públicas o privadas. 


\section{CONCLUSIONES}

En este estudio presentamos Saminbot, un chatbot para recolectar datos e informar a la población de la región del Cusco. La recolección de datos fue llevada a cabo a través de encuestas en las áreas de salud, economía y educación. Estas encuestas fueron preparadas y diseñadas bajo la asesoría de especialistas en las respectivas áreas, respetando la privacidad de los usuarios. La conversación de Saminbot con los usuarios busca ser personalizada según el tipo de usuario que interactúa con el chatbot (estudiantes, docentes o ciudadanos de la región del Cusco). Saminbot es un chatbot multi-plataforma que utiliza diferentes servicios de mensajería instantánea para el intercambio de mensajes. El chatbot es accesible mediante Whatsapp Messenger, Facebook Messenger y el propio chat del sitio web de Saminbot. Fueron recolectados 1586 registros para las tres áreas desde enero hasta junio del 2021. Estas interacciones fueron en su mayoría por pobladores de la provincia de Cusco, jóvenes y con nivel educativo universitario. En los cuestionarios de los sectores economía y educación, la mayoría fueron del género femenino. En este artículo se presentaron solo algunos resultados estadísticos descriptivos destacados sobre los datos debido a que el principal objetivo fue mostrar el potencial del uso de este tipo de herramientas tecnológicas como medio de recolección de datos en periodos de crisis como el vivido durante la pandemia del COVID-19. Sin embargo, los datos se encuentran publicados en repositorios para su posterior uso responsable.

Existe gran potencial para el uso de esta tecnología por instituciones públicas e interesados. No obstante, se mostraron limitaciones en el proyecto: (1) Las personas requieren de conexión a internet y estar familiarizados con la tecnología para poder interactuar con el chatbot, solo un porcentaje de la población tiene acceso a internet. (2) Los datos obtenidos pueden ser representativos para la provincia y no para la región Cusco. (3) No fue obligatorio que los individuos completen los cuestionarios de salud, educación y economía en su conjunto. Para responder los tres cuestionarios se requeriría de más tiempo.

\section{AGRADECIMIENTOS}

Agradecimiento a la Universidad Nacional de San Antonio Abad del Cusco (UNSAAC) por el soporte al proyecto en el año 2020. Agradecimiento a los especialistas que asesoraron el proyecto Dra. Regina Agramonte, Dra. Karen Mozo Velazco, Econ. Norma Vargas. 


\section{REFERENCIAS}

Adolph, C., Amano, K., Bang-Jensen, B., Fullman, N., y Wilkerson, J. (2021). Pandemic Politics: Timing State-Level Social Distancing Responses to COVID-19. Journal of Health Politics, Policy and Law, 46(2), 211-233. https://doi.org/10.1215/036168788802162

Bentlage, E., Ammar, A., How, D., Ahmed, M., Trabelsi, K., Chtourou, H., y Brach, M. (2020). Practical Recommendations for Maintaining Active Lifestyle During the COVID-19 Pandemic: A Systematic Literature Review. International journal of environmental research and public health, 17(17), 6265. https://doi.org/10.3390/ijerph17176265

Besolí, G., Palomas, N., y Chamarro, A. (2018). Uso del móvil en padres, niños y adolescentes: Creencias acerca de sus riesgos y beneficios. Aloma: Revista de Psicologia, Ciències de l'Educació i de l'Esport, 36(1), 29-39. https://doi. org/10.51698/aloma.2018.36.1.29-39

Bharti, U., Bajaj, D., Batra, H., Lalit, S., Lalit, S. , y Gangwani, A. (2020). Medbot: Conversational Artificial Intelligence Powered Chatbot for Delivering Tele-Health After COVID-19. 2020 5th International Conference on Communication and Electronics Systems (ICCES), 870-875. https://doi.org/10.1109/ICCES48766.2020.9137944

Borio, C. (2020). The COVID-19 Economic Crisis: Dangerously Unique. Business Economics, 55(4), 181-190. https://doi.org/10.1057/s11369-020-00184-2

Chatterjee, S. S., Malathesh Barikar, C., y Mukherjee, A. (2020). Impact of COVID-19 Pandemic on Pre-Existing Mental Health Problems. Asian Journal of Psychiatry, 51, 102071. https://dx.doi.org/10.1016\%2Fj.ajp.2020.102071

Ciotti, M., Ciccozzi, M., Terrinoni, A., Jiang, W.-C., Wang, C.-B., y Bernardini, S. (2020). The COVID-19 Pandemic. Critical Reviews in Clinical Laboratory Sciences, 57(6), 365-388. https://doi.org/10.1080/10408363.2020.1783198

Figueroa, T. A., Castro, J. M., Calderon, A. I. y Alburqueque, C. A. (2021). Escuelas rurales en el Perú: factores que acentúan las brechas digitales en tiempos de pandemia (COVID-19) y recomendaciones para reducirlas. Educación, 30(58). https://doi. org/10.18800/educacion.202101.001

Garcia Brustenga, G., Fuertes Alpiste, M., y Molas Castells, N. (2018). Briefing Ppaper: Los chatbots en educación. http://hdl.handle.net/10609/85786

Giraldo, E. B. (2020). COVID-19 in Peru. Indian Journal of Psychiatry, 62(Suppl 3), S498. https://dx.doi.org/10.4103\%2Fpsychiatry.IndianJPsychiatry_1045_20

GOB.PE. (2020). Plataforma nacional de datos abiertos [Recuperado la fecha 202109-21]. https://www.datosabiertos.gob.pe/ 
GOV.UK. (2020). COVID-19: Health Secretary's Sstatement to Parliament. https://www. gov.uk/government/speeches/COVID-19-health-secretarys-statement-toparliament

Guidon, A. C., y Amato, A. A. (2020). COVID-19 and Neuromuscular Disorders. Neurology, 94(22), 959-969. https://doi.org/10.1212/WNL.0000000000009566

Haug, N., Geyrhofer, L., Londei, A., Dervic, E., Desvars-Larrive, A., Loreto, V., Pinior, B., Thurner, S., y Klimek, P. (2020). Ranking the Effectiveness of Worldwide COVID-19 Government Interventions. Nature Human Behaviour, 4(12), 1303-1312. https:// doi.org/10.1038/s41562-020-01009-0

He, W., Zhang, Z., y Li, W. (2021). Information Technology Solutions, Challenges, and Suggestions for Tackling the COVID-19 Pandemic. International Journal of Information Management, 57, 102287. https://doi.org/https://doi.org/ 10.1016/j. ijinfomgt.2020.102287

Herrero-Diz, P., y Varona-Aramburu, D. (2018). Uso de chatbots para automatizar la información en los medios españoles. El profesional de la información (EPI), 27(4), 742-749. http://profesionaldelainformacion.com/contenidos/2018/jul/03.pdf

Hinchcliffe, D. (2016). How Chatbots and Artificial Intelligence are Evolving the Digital/ Social Experience. https://dionhinchcliffe.com/2016/04/13/how-chatbots-and-artifi cial-intelligence-are-evolving-the-digitalsocial-experience/

Io, H.. y Lee, C. (2017). Chatbots and Conversational Agents: A Bibliometric Analysis. 2017 IEEE International Conference on Industrial Engineering and Engineering Management (IEEM), 215-219. https://doi.org/10.1109/IEEM.2017.8289883

Jennings, R. B., Nahum, E. M., Olshefski, D. P., Saha, D., Shae, Z.-Y., y Waters, C. (2006). A Study of Internet Instant Messaging and Chat Protocols. IEEE Network, 20(4), 16-21. https://doi.org/10.1109/MNET.2006.1668399

Judson, T. J., Odisho, A. Y., Young, J. J., Bigazzi, O., Steuer, D., Gonzales, R., y Neinstein, A. B. (2020). Implementation of a Digital Chatbot to Screen Health System Employees During the COVID-19 Pandemic. Journal of the American Medical Informatics Association, 27(9), 1450-1455. https://doi.org/10.1093/jamia/ocaa130

Kaufmann, K., y Peil, C. (2020). The mobile instant messaging interview (mimi): Using Whatsapp to Enhance Self-Reporting and Explore Media Usage in situ. Mobile Media y Communication, 8(2), 229-246. https://doi.org/10.1177\%2F2050157919852392

Martin, A., Nateqi, J., Gruarin, S., Munsch, N., Abdarahmane, I., Zobel, M., y Knapp, B. (2020). An Artificial Intelligence-Based First-Line Defence Against COVID-19: Digitally Screening Citizens for Risks Via a Chatbot. Scientific reports, 10(1), 1-7. https://doi.org/10.1038/s41598-020-75912-x 
Mejía, D., Pallota, A., Egúsquiza, E., y Virreira Centellas, R. (2015). Encuesta de medición de capacidades financieras en los países andinos. Informe para Bolivia 2014. http://scioteca.caf.com/handle/123456789/741

Muhammad, A. F., Susanto, D., Alimudin, A., Adila, F., Assidiqi, M. H., y Nabhan, S. (2020). Developing English Conversation Chatbot Using Dialogflow. 2020 International Electronics Symposium (IES), 468-475. https://doi.org/10.1109/ IES50839.2020.9231659

Rosruen, N. y Samanchuen, T. (2018). Chatbot Utilization for Medical Consultant System. 2018 3rd Technology Innovation Management and Engineering Science International Conference (TIMES-iCON), 1-5. https://doi.org/10.1109/TIMES-iCON.2018.8621678

Schubel, L. C., Wesley, D. B., Booker, E., Lock, J., y Ratwani, R. M. (2021). Population subgroup differences in the use of a COVID-19 chatbot. NPJ digital medicine, 4(1), 1-3. https://doi.org/10.1038/s41746-021-00405-8

Shadmi, E., Chen, Y., Dourado, I., Faran-Perach, I., Furler, J., Hangoma, P., Hanvoravongchai, P., Obando, C., Petrosyan, V., Rao, K. D., y col. (2020). Health equity and COVID-19: Global perspectives. International journal for equity in health, 19(1), 1-16. https:// doi.org/10.1186/s12939-020-01218-z

Stieger, S., y Göritz, A. S. (2006). Using instant messaging for internet-based interviews. CyberPsychology y Behavior, 9(5), 552-559. https://doi.org/10.1089/cpb.2006.9.552

Tarkar, P. (2020). Impact of COVID-19 pandemic on education system. International Journal of Advanced Science and Technology, 29(9), 3812-3814. http://sersc.org/journals/ index.php/IJAST/article/view/16620

Unidad de Gestión Educativa Local - Puno. (2020). Aplicación de la encuesta a docentes y padres de familia sobre la estrategia "Yo aprendo en casa" - Programa Auditores Juveniles. https://ugelpuno.edu.pe/web/2020/12/07/aplicacionde-la-encuestaa-docentes-y-padres-de-familia-sobre-la-estrategia-yoaprendo-en-casaprograma-auditores-juveniles/

Varona, L., y Gonzales, J. R. (2021). Dynamics of the impact of COVID-19 on the economic activity of peru. Plos one, 16(1), e0244920. https://doi.org/10.1371/journal. pone. 0244920

Wozniak, A., Willey, J., Benz, J., y Hart, N. (2020). Covid impact survey. Chicago, IL: National Opinion Research Center. https://www.covid-impact.org/results 


\section{ANEXOS}

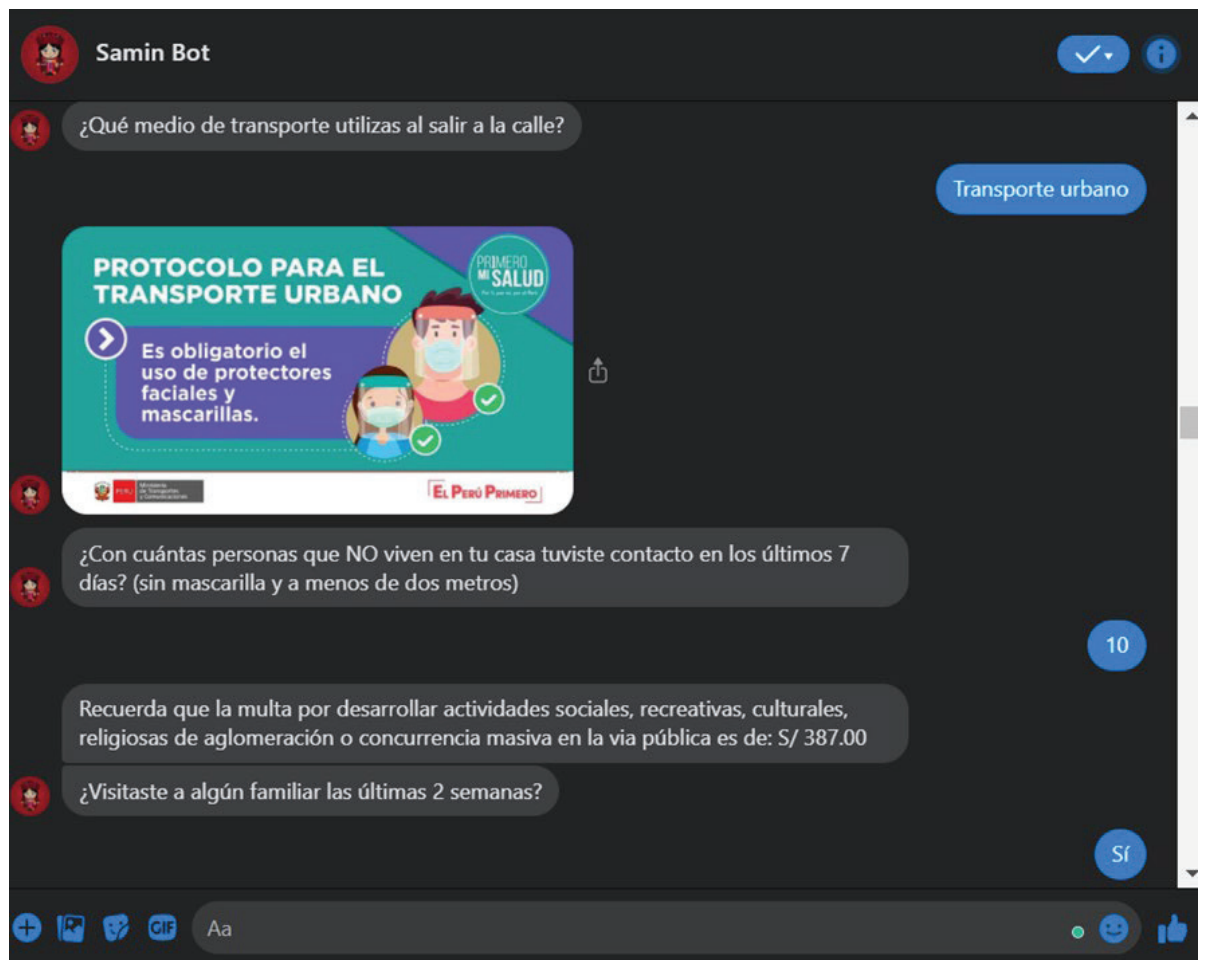

Figura 7. Evidencia de difusión de información - chatbot Salud

Fuente: Facebook 


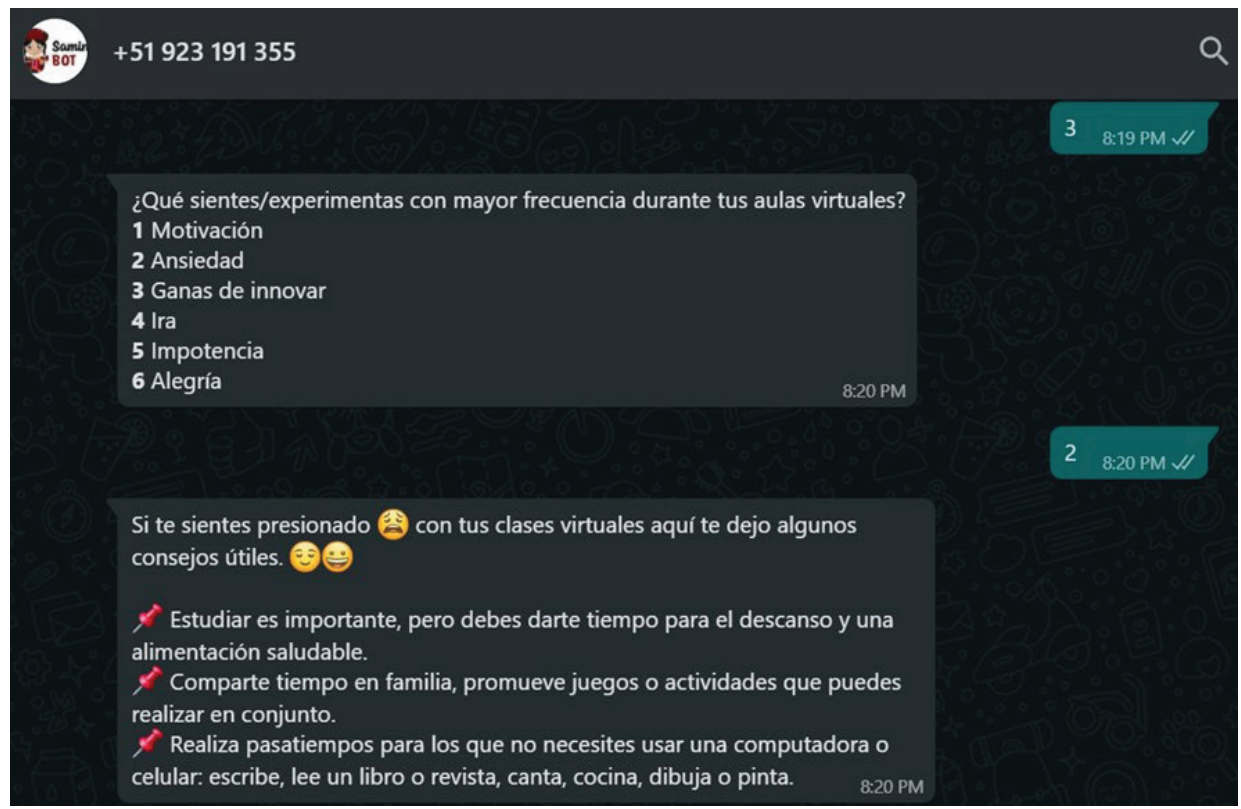

Figura 8. Evidencia de difusión de información - chatbot Educación

Fuente: WhatsApp

SaminBot

¿Su negocio tuvo alguno(s) de estos problemas financieros a causa de la pandemia del COVID-19? (Si tiene varias respuestas escribelas entre comas, por ejemplo: $1,2,5$ )

1 Remuneraciones del personal ŝ.

2 Compra de insumos o materia prima $\mathrm{ch}$.

3 Pago a proveedores $\hat{B}$.

4 Cobro a clientes है.

5 Limitaciones de acceso a financiamientos

6 Préstamos al sistema financiero 6

7 Ningunoe.

La politica de créditos de toda Entidad Financiera es especifica) Algunos de los

requisitos para documentos de empresas formales son 2 : RUC, balance, declaración

anual de IGV, impuesto a la renta, plan de inversión, otros.

Accede a información sobre créditos al final de la conversación.

¿Qué dificultades tuvo o tiene para conseguir un financiamiento para su negocio

durante la pandemia del COVID-19? (Si tiene varias respuestas escribelas entre comas

por ejemplo: $1,2,5$ )

1 Falta de garante.

2 Incapacidad de pago.

3 Falita de documentacion.

4 Sobrendeudamiento.

6 Ninguno de los anteriores

Figura 9. Evidencia de difusión de información - chatbot Economía

Fuente: WhatsApp 